\title{
Entrelacs
}

Cinéma et audiovisuel

\section{Avant-propos : Le cinéma ou les territoires de l'imaginaire}

\section{Françoise Marchand}

\section{(2) OpenEdition}

\section{Journals}

Édition électronique

URL : http://journals.openedition.org/entrelacs/225

DOI : 10.4000/entrelacs.225

ISSN : 2261-5482

Éditeur

Éditions Téraèdre

\section{Édition imprimée}

Date de publication : 1 février 2011

ISBN : 978-2-360850-03-7

ISSN : 1266-7188

\section{Référence électronique}

Françoise Marchand, « Avant-propos : Le cinéma ou les territoires de l'imaginaire », Entrelacs [En ligne], 8 | 2011, mis en ligne le 01 août 2012, consulté le 19 avril 2019. URL : http://journals.openedition.org/ entrelacs/225; DOl : 10.4000/entrelacs.225

Ce document a été généré automatiquement le 19 avril 2019

Tous droits réservés 


\title{
Avant-propos : Le cinéma ou les territoires de l'imaginaire
}

\author{
Françoise Marchand
}

1 Ce titre en forme d'hommage à Edgar Morin ${ }^{1}$ est une invitation à l'exploration d'un univers qui depuis 1895 ne cesse de questionner le rapport entre le réel et l'imaginaire.

Dans la préface à son ouvrage Edgar Morin écrit ${ }^{2}$ : «Ce qui m'avait sans cesse animé en travaillant L'Homme et la mort, c'était l'étonnement devant ce formidable univers imaginaire de mythes, dieux, esprits, univers non seulement surimprimé sur la vie réelle, mais faisant partie de cette vie anthroposociale réelle. C'était en somme l'étonnement que l'imaginaire soit partie constitutive de la réalité humaine. [...] Or, à sa façon, le formidable sentiment de réalité émanant des images artificiellement reproduites sur écran, me posait, comme à l'envers, le même problème. [...] Et je suis parti de cette question : dans quel sens et de quelle façon l'univers cinématographique moderne ressuscite-t-il l'univers archaïque des doubles? Pourquoi, le cinématographe, à l'origine une techniqu e de reproduction $\mathrm{du}$ mouvement dont l'usage semblait devoir être pratique, voire scientifique, a-t-il dès sa naissance, dérivé en cinéma, c'est-à-dire en spectacle imaginaire?»

Cette question du lien indéfectible entre réel et imaginaire n'a pourtant pas toujours été reconnue, et d'Epicure à Kant en passant par les stoïciens, Pascal, Leibniz... la question de l'imagination et de l'imaginaire a donné lieu à un grand nombre de théories. Depuis l'Antiquité classique jusqu'à Jung, l'histoire de l'imaginaire se confond avec celle de l'image et par là même avec celle des différents statuts que les sociétés ont bien voulu lui donner.

4 Depuis les travaux de Jung (sur l'image, les symboles et la notion d'archétype), puis grâce aux recherches de Gaston Bachelard ${ }^{3}$, Mircéa Eliade ${ }^{4}$, Henry Corbin ${ }^{5}$, Edgar Morin ${ }^{6}$ et ceux de Gilbert Durand ${ }^{7}$ la notion d'imaginaire perd enfin sa caractéristique de "folle du logis » si chère à Malebranche, et celle de chimère (Pascal, Voltaire, Rousseau) pour conquérir son véritable statut de moteur de la construction de l'esprit et en faire une fonction centrale de la psyché humaine, une fonction de création vitale ${ }^{8}$. 
5 C'est comme reproduction du réel que l'image a d'abord joué un rôle au cinéma. L'invention du cinéma est déjà en elle-même le résultat d'un imaginaire... l'aboutissement d'une réflexion issue du cerveau de nombreux rêveurs. La concrétisation, à des siècles d'intervalle, de la caverne de Platon.

6 Image d'Épinal, certes, mais jusqu'à preuve du contraire, personne n'a jamais rien trouvé de mieux pour montrer le côté à la fois réel et imaginaire de l'image projetée et la conscience d'être dans un univers qui, dès 1909, faisait dire à Apollinaire « Le cinéma est créateur d'une vie surréelle.» Cette notion sera reprise par André Breton en 1921 lorsqu'il écrit le Manifeste du surréalisme : «Je crois à la résolution future de ces deux états, en apparence si contradictoires, que sont le rêve et la réalité, en une sorte de réalité absolue, de surréalité, si l'on peut dire. »

7 Entre mythologie, rêve et sommeil le cinéma de Cocteau baigne dans les gouffres infinis de l'imaginaire. Dans son article Rana El Gharbie propose d'analyser la notion d'invisibilité dans l'œuvre cinématographique du poète. Comment ce dernier filme-t-il l'invisible ? Où mène-t-il le spectateur? Quel est son rôle quand il assiste au défilement des images de son imaginaire? Enfin, pourquoi Cocteau est-il hanté par la rencontre de l'invisible?

Lorsqu'il écrit Le cinéma ou l'homme imaginaire Edgar Morin poursuit : "L'image, ce n'est pas seulement la plaque tournante entre le réel et l'imaginaire, c'est l'acte constitutif radical et simultané du réel et de l'imaginaire. "

Entre le réel et l'imaginaire au cinéma il y a donc quelque chose. D'un côté l'être pensant, de l'autre un écran. Entre les deux : l'image. L'image et tout ce qu'elle comporte à la fois d'évidence mais également de trouble. L'image comme support de nos rêves, de nos fantasmes, comme intermédiaire entre notre univers et celui des dieux (stars ?). L'image, (reflet, mimesis, double, ...) comme réponse possible à nos interrogations.

C'est sur ces terres mouvantes où le corps de l'autre devient le lieu d'exploration ultime que s'est aventurée Claudine Le Pallec Marand. La question est posée dès le départ: l'imaginaire du féminisme est-il soluble dans la mise en scène ? Pour l'auteur, qui s'appuie sur une filmographie très dense, le cinéma de Quentin Tarantino est l'occasion d'affirmer que le féminisme n'est pas seulement un sujet thématique mais un enjeu de mise en scène : filmer les dialogues, filmer la hiérarchie des sexes, filmer la communauté, filmer la maîtrise du corps, filmer la séduction du corps...

11 La séduction mais aussi la peur de l'autre, du sexe de l'autre... Dans Teeth de Mitchell Lichtensten, l'héroïne, qui prône l'abstinence, est dotée d'un sexe "denté " prêt à émasculer les hommes mal intentionnés. Dans son article, Nathan Reneaud montre comment ce film qui n'est pas une œuvre de science-fiction au sens strict du terme (il se situe plutôt au confluent du Teen movie, du cinéma gore et de la SF) fonctionne comme arrière-plan fantasmatique, réservoir mythologique ou encore relais de peurs archaïques à l'endroit de la sexualité féminine. L'auteur va plus loin encore en précisant que ce rejet de l'altérité, cette peur de l'animalité n'est que le reflet de l'« éthique créationniste » qui travaille la SF made in USA.

12 Le cinématographe fut conçu pour étudier le mouvement: il devient le plus grand spectacle du monde moderne. L'appareil de prise de vues semblait destiné à calquer le réel : il se mit à fabriquer des rêves. L'écran paraissait devoir présenter un miroir à l'être humain : il offrit au Xx ${ }^{\text {ème }}$ siècle ses demi-dieux, les stars. ${ }^{9}$ 
Imaginer l'autre c'est essayer de se définir soi-même, précise Gaëlle Lombard dans son article. Dans la mesure où il pose le fait de traduire l'autre en images comme une nécessité, l'imaginaire est le territoire privilégié où se cristallise ce désir. Quel que soit le stade de sa formation (schème, archétype, mythe, symbole), l'image pousse l'autre à devenir l'objet et le sujet d'un regard.

14 Pour ce numéro d'Entrelacs, envisagé comme l'exploration d'un territoire dont les limites semblent infinies, les auteurs se sont faits explorateurs d'une histoire, d'une géographie... C'est à ce voyage que nous invite Philippe Ragel, en compagnie d'Abbas Kiarostami. Franchir un seuil nous dit l'auteur, oser une traversée pour un autre monde, passer à d'autres modalités d'être au monde. Chez Kiarostami le chemin se présente souvent comme une figure libératoire, douée d'une puissance dynamique jamais entamée, une figure de l'écart, de la bifurcation, partant de l'insubordination.

Le parcours sur cette carte de l'imaginaire au cinéma a permis des approches diverses dont celle de la technique n'est pas la moins surprenante. Emmanuelle Bobée propose, elle, un classement des effets sonores et musicaux dans le premier film de David Lynch, Eraserhead. Ce classement, qui repose à la fois sur la localisation de la source sonore et sur la détermination du point d'écoute, permet de rendre compte des procédés utilisés afin d'introduire une subjectivisation du récit filmique et de comprendre pourquoi le traitement sonore joue un rôle prépondérant dans la compréhension du film.

Pour Edgar Morin, la dialectique objectif-subjectif s'incarne dans différentes figures de style cinématographiques (la surimpression, le fondu...). Hélène Vally dans son article fait sienne cette théorie, et étudie le flou, qui, utilisé à son extrême, synthétise remarquablement bien les problématiques développées par Edgar Morin. En effet, pour l'auteur, le flou permet d'accroître la participation affective du spectateur, car celui-ci est invité à imaginer ce que cache le trouble des images.

Du flou à l'écran crevé, il n'y a qu'un pas que Philippe Morice franchit en analysant Portrait of Space, photographie prise en 1937 par Lee Miller dans l'oasis égyptienne de Siwa. Un châssis parallèle au plan de l'image soutient un pan de moustiquaire éventré. Et par cet espace ouvert sur l'espace, nos souvenirs viennent se précipiter. Loin d'être un vide, l'ouverture dans la moustiquaire devient l'écran d'un cinéma qui repasserait en continu nos fantasmes de mystère, de visages voilés, de corps dissimulés.

8 Quelle articulation est-il donc possible de mettre en place entre ces deux territoires si proches et si contradictoires que sont ceux du cinéma qui filme le réel et celui qui le recrée ? C'est ce à quoi s'attache Jessie Martin dans son article sur le cinéma d'animation. Elle nous parle ainsi de certains films qui installent leur diégèse dans des conditions de réalité les plus proches du monde physique pour conduire leurs personnages dans un territoire souvent contigu où règnent les lois de l'imaginaire. C'est la cœxistence et surtout l'articulation de ces deux territoires fictionnels mais déterminés par leur rapport au réel qu'interroge l'auteur.

Enfin, Roland Carrée propose de franchir un pas de plus en effet boomerang lorsque l'imaginaire « animé » s'efface au profit d'un imagin aire « humanimé ». L'auteur évoque le film Wall.E (des studios Pixar) qui propose une rupture avec les films d'animation précédents en se posant comme film hybride dans lequel l'image du réel, celle d'êtres humains de chair et d'os appartenant au passé, s'affiche au sein même de l'image animée, celle du petit robot et de son univers futuriste. Roland Carrée précise que le film travaille le spectateur selon un niveau inverse, qui consiste à démontrer qu'à force de dévouer son 
imagination à l'image de synthèse, cette dernière en viendra petit à petit à remplacer le réel.

L'imagination, comme son territoire l'imaginaire, est le plus grand espace de liberté encore à la disposition des êtres humains. Si grande est cette liberté qu'un cinéaste a prêté à un des ses personnages, pourtant issu lui-même de l'imagination des hommes, un imaginaire émouvant. L'effet boomerang, évoqué dans l'article précédent, trouve dans Les Ailes du désir de Wim Wenders un terrain d'expérimentation inédit. Deux territoires et un ange qui hésite entre sa mission céleste et sa fascination pour la vie terrestre. Dans ce film, le cinéaste nous parle du Berlin encore divisé de 1987, de cette tentation de l'ailleurs, et Françoise Marchand, de ce besoin qu'ont les êtres humains et les anges d'imaginer le bonheur.

«Avec l'image, le bonheur est toujours au bout des images! $»^{10}$

\section{NOTES}

1. Edgar Morin, Le cinéma ou l'homme imaginaire, Paris, Les éditions de Minuit, 1956.

2. Ibid

3. Notamment les cinq ouvrages consacrés aux éléments.

4. Entre autres: Traité d'histoire des religions, Paris, Payot, 1949 - Images et symboles, Paris, Gallimard, 1952 - Mythes, rêves et mystères, Paris, Gallimard, 1957.

5. Henry Corbin, L'imagination créatrice dans le soufisme d'Ibn'Arabî, Paris, Flammarion, 1976.

6. Edgar Morin, cf note 1 et Les stars, Paris, Le Seuil, 1957

7. Gilbert Durand, Les structures anthropologiques de l'imaginaire, Paris, 1960 (Dunod, 1992) L'imagination symbolique, Parfis, PUF, Quadrige, 1993

8. Même si Sartre ramène l'imagination à un jugement de négation : imaginer, c'est poser l'objet comme n'étant pas là (L'imaginaire, Paris, Gallimard, 1940 - L'imagination, Paris, PUF, 1950) et que pour Lacan l'imaginaire est le signe d'un échec de la fonction symbolique de l'être humain (Le stade du miroir comme formateur de la fonction du Je, Écrits, Paris, Seuil, 1966) il est important de rappeler que chez ce même être humain, comme chez les animaux, interrompre la phase du rêve durant le sommeil conduit à la mort.

9. Edgar Morin, Les stars, Paris : Editions du Seuil, 1957.

10. Serge Tisseron, Psychanalyse de l'image, Paris, Dunod, 2005.

\section{AUTEUR}

\section{FRANÇOISE MARCHAND}

Maître de Conférences

Université de Toulouse II le Mirail 